\title{
Synthesis of reflection gratings for advanced plasma heating scenarios
}

\author{
$\underline{B . ~ P l a u m}^{1}$, M. Schubert ${ }^{2}$, A. Zeitler ${ }^{1}$, W. Kasparek ${ }^{1}$, C. Lechte ${ }^{1}$, J. Stober $^{2}$ and \\ ASDEX Upgrade Team \\ ${ }^{1}$ Institut für Grenzflächenverfahrenstechnik und Plasmatechnologie, Universität Stuttgart, Pfaffenwaldring 31, \\ D-70569 Stuttgart, Germany, plaum@igvp.uni-stuttgart.de \\ ${ }^{2}$ Max-Planck-Institut für Plasmaphysik, Boltzmannstr.2, D-85748 Garching, Germany
}

When heating fusion plasmas at higher harmonics of the electron cyclotron frequency, the absorption efficiency can be reduced. This leads to a significant transmitted beam power hitting the wall at the high field side (HFS).

To protect the wall and to use the remaining power, one method is to place a specialized reflector at the HFS, which directs the beam back into the plasma for a second heating pass [1]. The reflector needs to conform to the tiles at the HFS, which are often nonplanar. The direction of the reflected beam is chosen such that the absorption of the second heating pass is maximized and the wall area at the LFS, which is hit after the second pass, contains no sensitive components. Additional requirements are a refocusing of the beam and polarization independence of the reflection characteristics. All these conditions can only be fulfilled with a grating. The design process involves the decomposition of the $3 \mathrm{D}$ field problem into a series of $2 \mathrm{D}$ reflections of plane waves. After the $2 \mathrm{D}$ gratings are optimized, the final 3D grating is synthesized.

In earlier works [1], [2] a code for the optimization of such gratings was already developed and several tiles were successfully designed. This code was modernized and a number of new features were added.

The incident and reflected beams are defined in terms of astigmatic Gaussian beam parameters and the orientations of the beam axes. Together with the basic shape of the reflector (e.g. saddle-type for the HFS of a tokamak) local k-vectors $\mathbf{k}_{\text {in }}$ and $\mathbf{k}_{\text {out }}$ can be calculated for every point on the mirror. This $3 \mathrm{D}$ problem can now be described by an ordinary reflection parallel to the groove and the diffraction of a plane wave at an infinite grating perpendicular to the groove.

$$
\text { Grooves }
$$

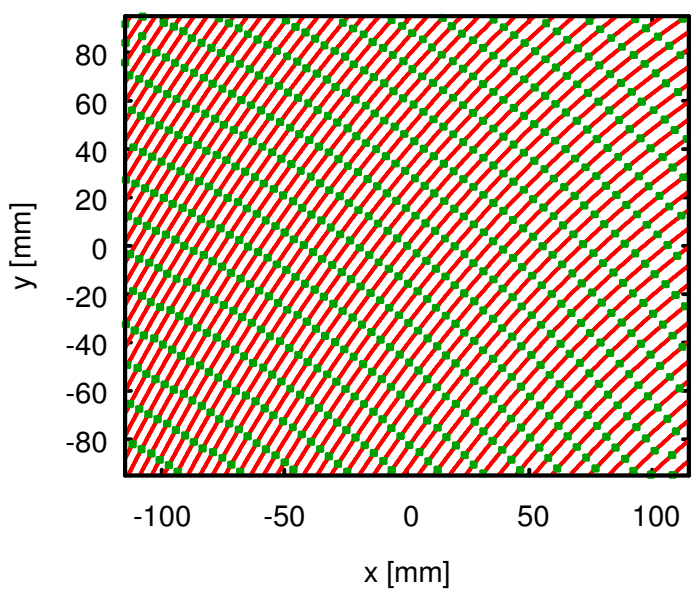

Fig. 1. Groove layout (lines) and optimization points (dots) for the $2 \mathrm{D}$ profiles
The only parameters describing the $2 \mathrm{D}$ diffraction are the beam angles $\Theta_{\text {in }}$ and $\Theta_{\text {out }}$ and the diffraction order $n$. Since the direction of the grooves can be calculated from $\mathbf{k}_{\text {in }}, \mathbf{k}_{\text {out }}$ and the normal vector, the whole groove layout can be obtained by a simple Runge Kutta integration of the grating vectors. Along the grooves, we define the points, for which a $2 \mathrm{D}$ profile will be optimized (see Fig. 1).

The goal of the optimization is to maximize the reflected power in the desired order for both polarizations and to minimize the phase shift between the polarizations. The minimum phase shift is necessary to preserve an incident elliptical polarization, which is required for current-drive scenarios.

The profile of a single groove is described by Fourier coefficients, where the maximum order can be obtained with the sampling theorem from the ratio of the groove period to the wavelength.

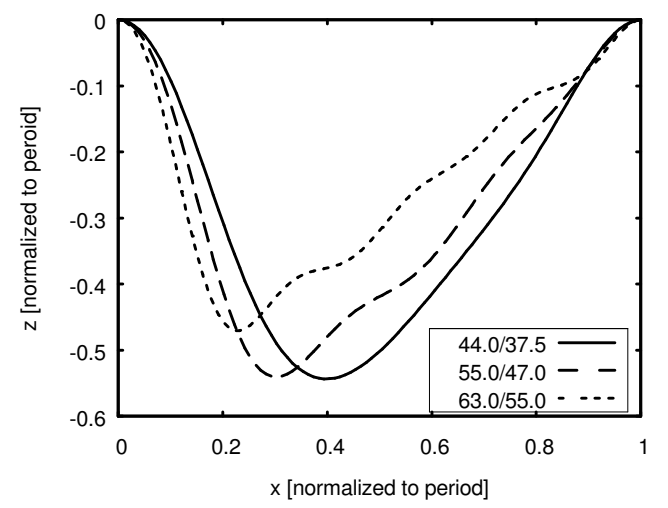

Fig. 2. Optimized 2D profiles for different combinations of $\Theta_{\text {in }}$ and $\Theta_{\text {out }}$

Fig. 2 shows optimized 2D profiles for different combinations of $\Theta_{\text {in }}$ and $\Theta_{\text {out }}$. The grooves are shifted such, that the highest points are at the interval boundaries. This ensures a smooth transition between adjacent grooves.

The final 3D grating is then synthesized by interpolation, Fig. 3 shows the scheme. The cross is the point $(x, y)$, for which the local $z$-coordinate is calculated. The first step is to identify the groove. Then, the mirror coordinates are transformed into groove local coordinates $(u, v)$. The Fourier coefficients are then obtained from the nearest optimized profiles by a simple 1D interpolation in the direction of the $v$ coordinate.

Fig. 4 shows the final interpolated grating of a reflector, which was designed for the ECRH-2 system of ASDEX-Upgrade [3]. It resembles the groove layout from Fig. 1 and shows also the saddle type shape of the reflector. The reflector was manufactured from 
a graphite blank using an NC milling machine (See Fig. 5).

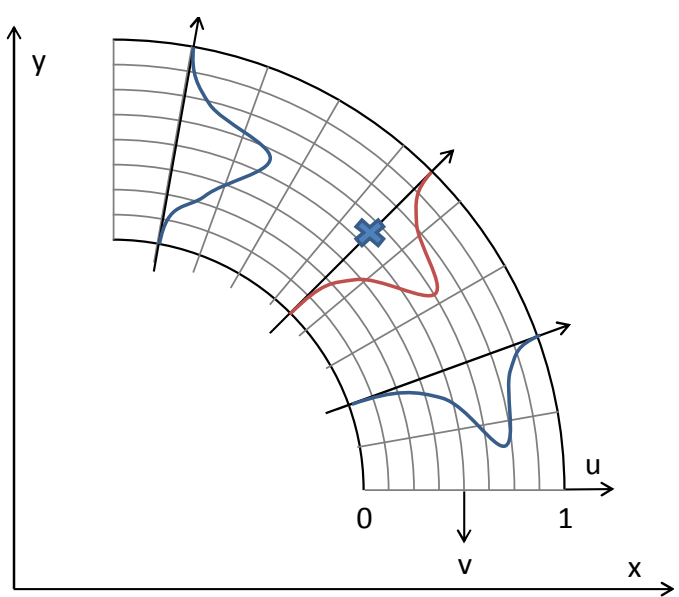

Fig. 3. Interpolation scheme for the final $3 \mathrm{D}$ grating. Note that in general the groove width is not constant

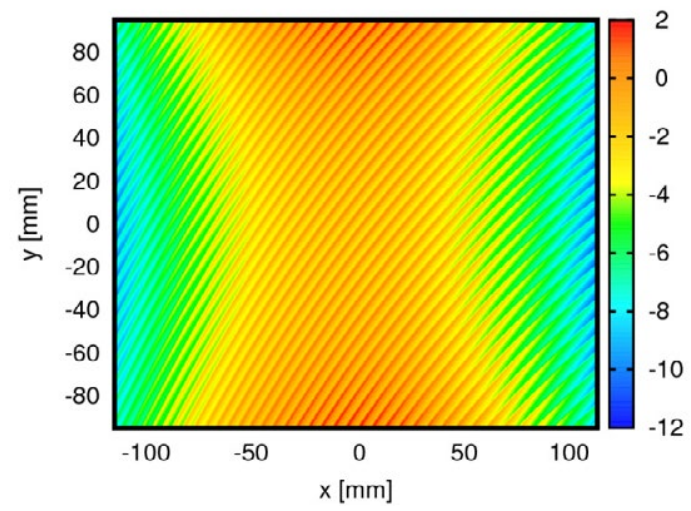

Fig. 4. Interpolated grating

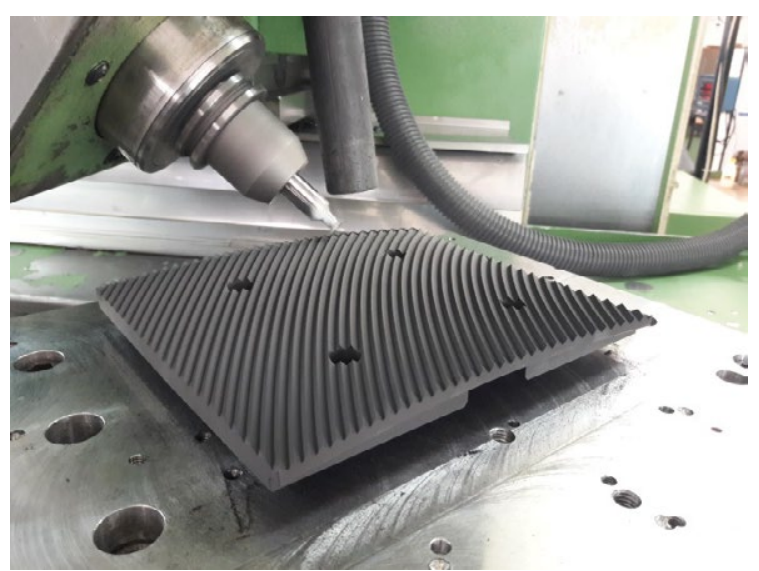

Fig. 5. Reflector in the milling machine

For an experimental characterization the reflector was mounted on a goniometer and illuminated by a horn antenna with a Gaussian beam. The reflected field was measured with an $\mathrm{x}-\mathrm{y}$ scanner and a vector network analyzer. The measured intensity and phase are shown in Figs. 6 and 7. One can see a nicely focused beam and an astigmatic phase similar to the design value.
The reflector was coated with a thin tungsten layer $(13-15 \mu \mathrm{m})$ to conform to the ASDEX-Upgrade requirements and is already installed in the tokamak. High power tests will be performed during the next experimental campaign.

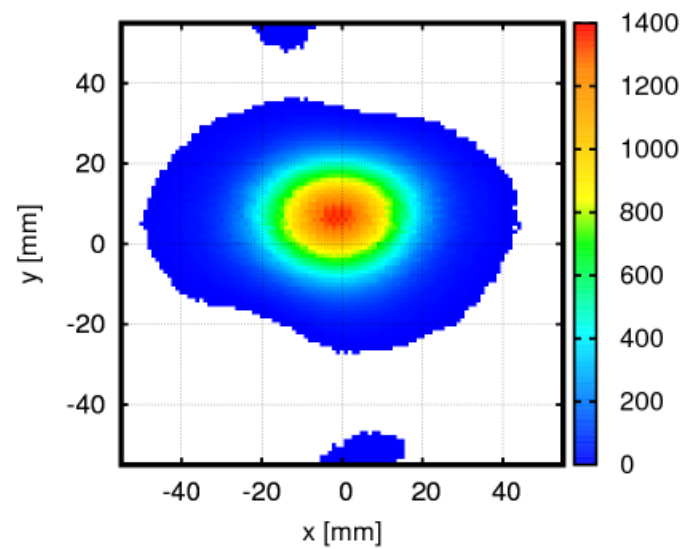

Fig. 6. Measured intensity for horizontal polarization

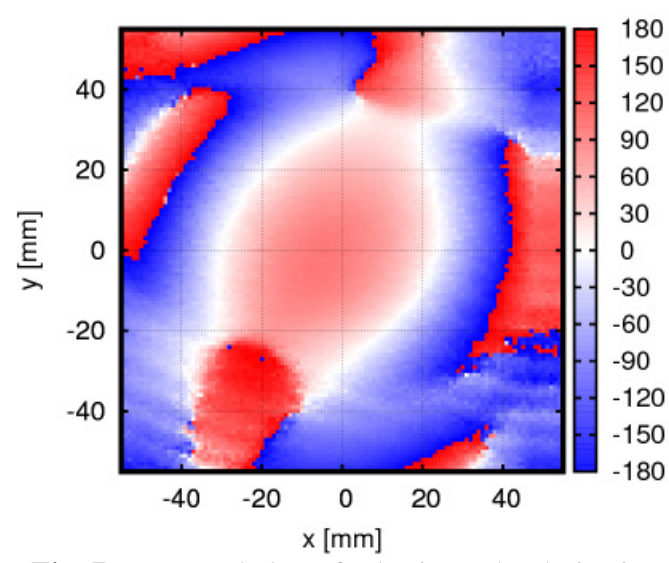

Fig. 7. Measured phase for horizontal polarization

\section{References}

1. H. Höhnle, J. Stober, A. Herrmann, W. Kasparek, F. Leuterer, F. Monaco, R. Neu, D. Schmid-Lorch, H. Schütz, J. Schweinzer, U. Stroth, D. Wagner, S. Vorbrugg, E. Wolfrum and the ASDEX Upgrade Team, Extension of the ECRH operational space with $\mathrm{O} 2$ and X3 heating schemes to control tungsten accumulation in ASDEX Upgrade, Nucl. Fusion, 51, 083013 (2011)

2. O. Mangold, Effiziente holographische Gitterspiegel für die Elektronzyklotronresonanzheizung von Fusionsplasmen, Doctoral Thesis, Universität Stuttgart, 2009

3. D. Wagner, J. Stober, F. Leuterer, F. Monaco, S. Müller, M. Münich, C. J. Rapson, M. Reich, M. Schubert, H. Schütz, W. Treutterer, H. Zohm, M. Thumm, T. Scherer, A. Meier, G. Gantenbein, J. Jelonnek, W. Kasparek, C. Lechte, B. Plaum, T. Goodman, A.G. Litvak, G.G. Denisov, A. Chirkov, V. Zapevalov, V. Malygin, L.G. Popov, V. O. Nichiporenko, V. E. Myasnikov, E. M. Tai, E.A. Solyanova, S.A. Malygin, ASDEX Upgrade Team, Status, Operation, and Extension of the ECRH System at ASDEX Upgrade, Journal of Infrared, Millimeter, and Terahertz Waves, 37, 45-54 (2016) 